\title{
Particle transfer in edge transport barrier with stochastic magnetic field
}

M. Z. Tokar, T. E. Evans, R. Singh, and B. Unterberg

Citation: Physics of Plasmas 15, 072515 (2008); doi: 10.1063/1.2959122

View online: https://doi.org/10.1063/1.2959122

View Table of Contents: http://aip.scitation.org/toc/php/15/7

Published by the American Institute of Physics

\section{Articles you may be interested in}

Plasma transport in stochastic magnetic field caused by vacuum resonant magnetic perturbations at diverted tokamak edge

Physics of Plasmas 17, 102503 (2010); 10.1063/1.3487733

The physics of edge resonant magnetic perturbations in hot tokamak plasmas

Physics of Plasmas 13, 056121 (2006); 10.1063/1.2177657

Plasma currents induced by resonant magnetic field perturbations in tokamaks

Physics of Plasmas 16, 042317 (2009); 10.1063/1.3126548

Changes in particle transport as a result of resonant magnetic perturbations in DIII-D

Physics of Plasmas 19, 056503 (2012); 10.1063/1.4718316

The interaction of resonant magnetic perturbations with rotating plasmas

Physics of Fluids B: Plasma Physics 3, 644 (1991); 10.1063/1.859863

Particle transport and rotation damping due to stochastic magnetic field lines

Physics of Fluids B: Plasma Physics 4, 1152 (1992); 10.1063/1.860123

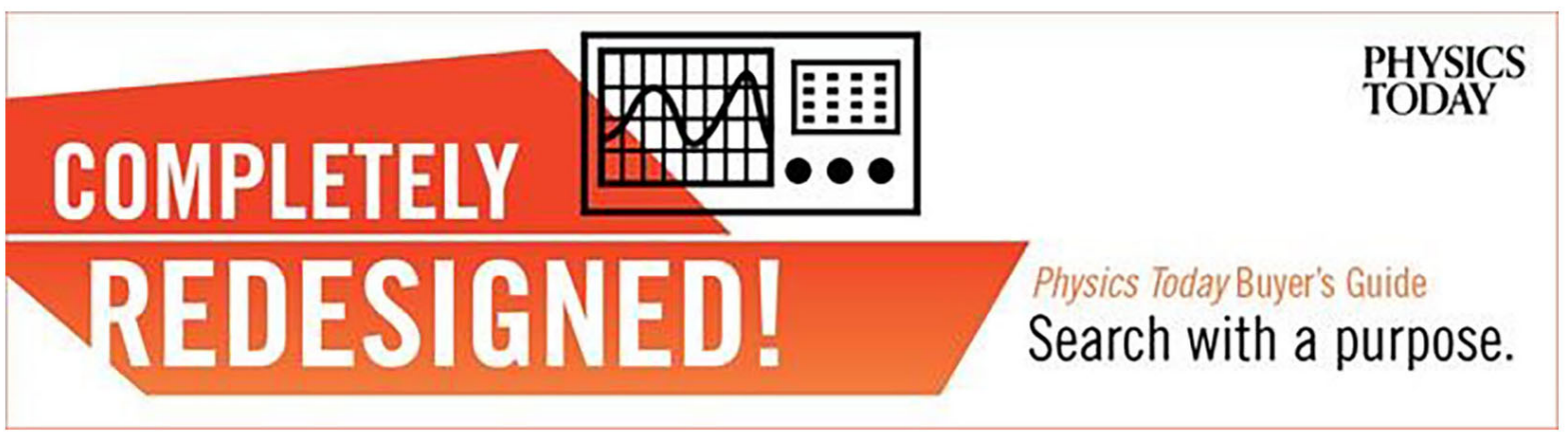




\title{
Particle transfer in edge transport barrier with stochastic magnetic field
}

\author{
M. Z. Tokar, ${ }^{1}$ T. E. Evans, ${ }^{2}$ R. Singh ${ }^{3}$ and B. Unterberg ${ }^{1}$ \\ ${ }^{1}$ Forschungszentrum Jülich GmbH, Institute of Energy Research IEF-4: Plasma Physics, \\ Association FZJ-Euratom, 52425 Jülich, Germany \\ ${ }^{2}$ General Atomics, San Diego, California 92186-5606, USA \\ ${ }^{3}$ Institute for Plasma Research, Bhat, Gandhinagar-382428, India
}

(Received 4 April 2008; accepted 25 June 2008; published online 25 July 2008)

\begin{abstract}
Charged particle losses at the plasma edge affected by resonant magnetic perturbations (RMP) are considered by taking into account the electron and ion flows both parallel and perpendicular to perturbed field lines. Calculations are done for H-mode plasmas of low collisionality, i.e., under conditions where significant pump out of particles has been observed in experiments on the DIII-D tokamak [J. Luxon, Nucl. Fusion 42, 614 (2002)] with RMP from the I-coils. It is demonstrated that the perpendicular ion flux, arising by magnetic field stochastization due to the deviation of poloidal rotation from the neoclassical one, is of more importance than the parallel ion flow. With both loss contributions included, computations provide a pump out level in agreement with observations if the screening of RMP by the plasma rotation is taken into account. The impact of possible enhancement in the perpendicular electron transport due to fluctuations observed with RMP in the edge transport barrier is assessed. (C) 2008 American Institute of Physics. [DOI: 10.1063/1.2959122]
\end{abstract}

\section{INTRODUCTION}

Significant pump out of plasma particles induced by external resonant magnetic perturbations (RMP) has been observed in several devices. ${ }^{1-3}$ This phenomenon normally accompanies suppression or mitigation of type I edge localized modes (ELM) in the presence of RMPs in collisionless H-mode plasmas. ${ }^{2,3}$ The fact that stronger density drop is seen by the total suppression of ELMs ${ }^{2}$ than by incomplete ELM mitigation ${ }^{3}$ reveals pump out as one of the key processes leading to the ELM modification with RMPs. It is normally believed that such a modification takes place if RMPs result in stochastization of magnetic field. ${ }^{2}$ In the previous analysis, ${ }^{4,5}$ the flows of electrons and ions along stochastic field lines were assumed ambipolar and considered as the main channel for the enhanced particle exhaust. The reduction of plasma density experimentally found in the DIII-D tokamak operated with the so called I-coils ${ }^{2}$ was reproduced well in calculations provided the RMP are not screened by plasma rotation ${ }^{6,7}$ in the Edge Transport Barrier (ETB).

Estimates show that the latter assumption should indeed be justified if the toroidal rotation only is taken into account. However, due to very sharp gradients of parameters, the poloidal plasma velocity in the ETB may be comparable with the toroidal velocity and, therefore, be more effective for the screening. It is demonstrated in this paper that in such a case, the parallel particle flows are not efficient enough to explain the experimental level of pump out, and other mechanisms for particle transfer in a stochastic magnetic field have to be taken into account.

In static plasmas with open stochastic field lines, quasineutrality requires the ambipolarity of radial fluxes of electrons and ions. If only flows along stochastic field lines contribute to radial particle losses, ${ }^{4,5}$ these flows have to be ambipolar also. The latter requirement can be satisfied only if the radial electric field $E_{r}$ is equal to a particular value $E_{\text {amb }}$ governed by the radial gradients of the electron density $n$ and temperature $T_{e}{ }^{8,9}$ On the other hand, by acting on ions, $E_{r}$ competes with the Lorentz force that arises due to the plasma rotation. Therefore, $E_{r}$ and the ion poloidal velocity $V_{\vartheta}$ are interdependent. If $E_{r}$ differs from $E_{\text {neo }}$ for which $V_{\vartheta}$ is equal to the velocity prescribed by neoclassical theory, a poloidal viscous force is generated leading to an outward drift of ions. ${ }^{10,11}$ The quasineutrality of the plasma is maintained owing to the deviation of $E_{r}$ from $E_{\text {amb }}$ thus allowing a parallel electron flux that exceeds the parallel ion one. Thus, in a stochastic region both parallel and perpendicular flows of electrons and ions can be generated that are nonambipolar apart.

In this paper, both parallel and perpendicular flows arising in a stochastic magnetic field are taken into account by considering the modifications in the density profile caused by the I-coils in DIII-D. ${ }^{2,12,13}$ The parallel flow is calculated from the plasma parallel motion equation as it is described in Ref. 5. The perpendicular flux is determined by taking into account the radial and poloidal force balances for ions and ambipolarity condition for the total radial fluxes. The RMP screening factor has been taken from Ref. 7, where it was calculated numerically on the basis of kinetic modeling relevant for the collisionless plasma in the ETB. The poloidal rotation velocity is estimated self-consistently while the toroidal flow is taken from experimental measurements of carbon ions that are assumed to be representative for the deuterium ion flow. ${ }^{12}$

\section{CHARACTERISTICS OF STOCHASTIC MAGNETIC FIELD}

Normally, an external magnetic perturbations is a superposition of Fourier harmonics $\widetilde{\mathbf{B}}_{M, N} \exp (i M \vartheta-i N \varphi)$ with diverse multiplicities $M$ and $N$ in the poloidal and toroidal 
directions $\vartheta$ and $\varphi$, respectively. At resonant magnetic surfaces (RMS) with the safety factor $q$ equal to the ratio $M / N$, an RMP produces magnetic islands with a width of ${ }^{14}$

$$
W_{M, N}=4 \sqrt{b_{M, N} R r /(N \hat{s})},
$$

where $b_{M, N}=\widetilde{B}_{M, N}^{r} / B, r$ and $R$ are the RMS minor and major radii, correspondingly, and $\hat{s}=d \ln q / d \ln r$ is the magnetic shear. If the perturbation is strong enough and the so called Chirikov parameter $\sigma_{\mathrm{Ch}}=W_{M, N} / \Delta r$, with $\Delta r=r /(M \hat{s})$ being the distance between adjacent RMS for which $\Delta q=1 / N$, exceeds 1 , different island chains are overlapped and magnetic field stochastization takes place. In this case, the behavior of field lines can be characterized by considering the distance between two neighboring field lines, $\delta$, which increases with the distance $l$ along the lines. Thus, $\delta$ grows up exponentially for small distances $l, \delta(l) \sim \exp \left(l / L_{K}\right)$, where $L_{K}$ is the socalled Kolmogorov length: ${ }^{1}$

$$
L_{K} \approx \pi q R\left(2 / \pi / \sigma_{\mathrm{Ch}}\right)^{4 / 3} .
$$

For $l \gg L_{K}$, field lines displace radially in a diffusive manner, $\delta(l)=\sqrt{2 D_{\mathrm{FL}} l}$. In a quasilinear approximation, the diffusivity $D_{\mathrm{FL}}$ is represented by the sum of Dirac functions centered on RMS, ${ }^{15} D_{\mathrm{FL}} \approx \pi q R \Sigma_{M, N} b_{M, N}^{2} \delta(M / q-N)$. In order to proceed to a smooth radial dependence of $D_{\mathrm{FL}}$, we assume that due to the broadening of resonances, the contribution from the $(M, N)$ harmonic is distributed over the whole island produced by it. Consequently, at a given radial position the sum has to include all harmonics whose islands comprise the point in question. If their amplitudes are not very different, the total number of such harmonics is equal to $\sigma_{\mathrm{Ch}}$ and

$$
D_{\mathrm{FL}} \approx \pi q R b_{M, N}^{2} \sigma_{\mathrm{Ch}} .
$$

Henceforth, we will use the formula above, although it was not rigorously derived yet.

The variation of $b_{M, N}$ with the distance from the separatrix at $r=a$ can be described in the first approximation by the following relation:

$$
b_{M, N}(r) \approx b_{M, N}^{0}(a) \times(r / a)^{\bar{M}-1} \times f_{\text {scr }} .
$$

Here $b_{M, N}^{0}(a)$ is the amplitude at the separatrix computed in vacuum approximation. The second factor is a geometrical one and $\bar{M}=M$ in a cylindrical approximation. ${ }^{16}$ In DIII-D with divertor, $\bar{M}<M$ since the poloidal mode number "seen" by the plasma is different from that of the I-coil located at the low field side where the pitch angle is larger than in the vicinity of the X-point. Firmly $\bar{M}$ and its radial variation can be determined only by calculating vacuum RMP numerically, see Refs. 17 and 18. Presently we neglect the difference between $\bar{M}$ and $M$ because this affects the radial behavior of $b_{M, N}$ much weaker than the last screening factor in Eq. (4), $f_{\text {scr. }}$. This takes into account the shielding of the RMP by currents induced on the resonant surface as a plasma response to perturbations. The estimation of the screening factor is a very delicate point. As it is demonstrated in Ref. 19, at least 10 different regimes of RMP shielding can be distinguished depending on the relations between characteristic times of different physical processes involved in the screen- ing phenomenon. Therefore, a numerical approach is the most reliable by determining $f_{\text {scr }}$, especially by taking into account that the MHD approximation applied in Ref. 19 may be questionable under collisionless conditions in question. Henceforth we use the results of Ref. 7, where the RMP shielding was numerically analyzed on the basis of a kinetic model. The radial variation of $f_{\text {scr }}$ computed for the plasma conditions in DIII-D similar to those discussed in the present paper are taken from Fig. 5 of Ref. 7.

\section{PARTICLE FLOWS IN STOCHASTIC MAGNETIC FIELD}

Ambipolarity constraint. In a stochastic field, radial gradients of plasma parameters generate fluxes of electrons and ions both perpendicular and parallel to the field lines, $\boldsymbol{\Gamma}_{\|}^{e, i}$ and $\boldsymbol{\Gamma}_{\perp}^{e, i}$, respectively. They have components in the radial direction normal to unperturbed magnetic surfaces, $\Gamma_{\|, r}^{e, i} \equiv \boldsymbol{\Gamma}_{\|}^{e, i} \cdot \widetilde{\mathbf{B}}_{r} / B$ and $\Gamma_{\perp, r}^{e, i} \equiv \boldsymbol{\Gamma}_{\perp}^{e, i} \cdot \mathbf{e}_{r}$, where $\mathbf{e}_{r}$ is the unit radial vector. Here, plasma quasineutrality is maintained when the ambipolarity constraint

$$
\Gamma_{r} \equiv \Gamma_{\|, r}^{i}+\Gamma_{\perp, r}^{i}=\Gamma_{\|, r}^{e}+\Gamma_{\perp, r}^{e}
$$

is satisfied for the flux components averaged over $\vartheta$ and $\varphi$.

Parallel flux contribution. The ion flow along stochastic field lines has been theoretically analyzed, e.g., in Refs. 20-22. It was assumed there that due to anomalous perpendicular viscosity, the parallel plasma flow is deeply subsonic and the nonlinear inertia term in a parallel momentum equation can be neglected compared to the pressure gradient. This assumption fails for the ETB conditions where the viscosity is at very low neoclassical level and radial gradients of plasma parameters are extremely sharp. Therefore, in Ref. 4 the viscous term was neglected but the inertia term has been retained. This approach was developed further in Ref. 5, where the radial variation of the field line inclination angle was taken into account. Henceforth, $\Gamma_{\|, r}^{i}$ is calculated by following Ref. 5.

According to Ref. 8, for the collisionless ETB in question

$$
\Gamma_{\|, r}^{e}=-n V_{\mathrm{th}, e} D_{\mathrm{Fl}}\left(\frac{d \ln n}{d r}+\frac{1}{2} \frac{d \ln T_{e}}{d r}+\frac{e E_{r}}{T_{e}}\right),
$$

where $V_{\text {th, } e}=\sqrt{2 T_{e} /\left(\pi m_{e}\right)}$ is the electron thermal velocity. Thus the ambipolarity of parallel electron and ion flows, $\Gamma_{\|, r}^{e}=\Gamma_{\|, r}^{i}$, is achieved when $E_{r}$ is equal to

$$
E_{\mathrm{amb}}=-\frac{T_{e}}{e}\left(\frac{\Gamma_{\|, r}^{i}}{n V_{\mathrm{th}, e} D_{\mathrm{Fl}}}+\frac{d \ln n}{d r}+\frac{1}{2} \frac{d \ln T_{e}}{d r}\right) .
$$

Consideration for ion parallel flow ${ }^{4,5}$ provides as a crude estimate $\Gamma_{\| r}^{i} \approx-c_{s} D_{\mathrm{Fl}} d n / d r$ with the ion sound velocity $c_{s}=\sqrt{\left(T_{i}+T_{e}\right) / m_{i}}$. Thus the first term in the brackets in Eq. (7) is smaller by a factor $\sqrt{m_{e} / m_{i}}$ than the other ones and $E_{\text {amb }}>0$ for normally negative gradients of density and temperature.

Perpendicular flux contribution. This flux component, $\Gamma_{\perp, r}^{i}$, is determined from the radial component of the ion force balance, ${ }^{10}$ 


$$
e E_{r}+e\left(V_{\vartheta} B_{\varphi}-V_{\varphi} B_{\vartheta}\right)-\frac{1}{n} \frac{d n T_{i}}{d r}=0
$$

and the equation

$$
\sqrt{\frac{\pi}{2} T_{i} m_{i}} \frac{q}{R} n\left(V_{\vartheta}-V_{\text {neo }}\right)=-e B \frac{\Gamma_{\perp, r}^{i}-\Gamma_{\perp, r}^{e}}{c}
$$

that follows from Eqs. (2.10) and (2.13) of Ref. 10 for collisionless plasmas. The left-hand side can be interpreted as a poloidal viscous force arising if the plasma rotation velocity deviates from the neoclassical one, $V_{\text {neo }}=k_{\text {neo }} / e B_{\varphi} \times d T_{i} / d r$, with the factor $k_{\text {neo }}$ depending on the inverse aspect ratio $\varepsilon=r / R$ and ion collisionality $\nu_{i *}=q R \varepsilon^{-3 / 2} / \lambda_{i}$ with $\lambda_{i}$ being the mean free path length between ion Coulomb collisions,

$$
k_{\text {neo }}=\frac{1.17-0.35 \nu_{i *}^{1 / 2}-2.1 \nu_{i *}^{2} \varepsilon^{2}}{1+0.7 \nu_{i *}^{1 / 2}+\nu_{i *}^{2} \varepsilon^{2}} .
$$

At the barrier top, the ion collisionality is of $0.03-0.1$, i.e., ions are in the banana regime and $k_{\text {neo }}=1.17$. The deviation of $V_{\vartheta}$ from $V_{\text {neo }}$ is predicted by neoclassical theory to lead to a poloidal force that can be expressed as either the left-hand side (LHS) or the right-hand side (RHS) of Eq. (9); see Eqs. (2.10) and (2.13) in Ref. 10, respectively. The RHS of Eq. (9) corresponds to a $j_{r} \times B$ torque induced by a radial current (do not mix with the "Fitzpatrick torque," which is essentially a $j_{\|} \times B_{r}$ one). Equation (9) thus implies that the deviation of $V_{\vartheta}$ from $V_{\text {neo }}$ leads to a radial ion current $\Gamma_{\perp, r}^{i}$ exceeding $\Gamma_{\perp, r}^{e}$. This is the key physics of our model. Unlike Eq. (2.13) in Ref. 10, also the electron current $\Gamma_{\perp, r}^{e}$ is retained in Eq. (9). This guarantees that even without RMP when $V_{\vartheta}=V_{\text {neo }}$ and ion and electron perpendicular currents are ambipolar, $\Gamma_{\perp, r}^{i}=\Gamma_{\perp, r}^{e}$, Eq. (9) remains valid. $\Gamma_{\perp, r}^{e}$ would appear in Eq. (2.13) of Ref. 10 if friction between electron and ions would not be neglected; see the remark at the bottom of p. 167 in Ref. 10. In this case, $\Gamma_{\perp, r}^{e}$ is a neoclassical one. Since drift motion induced by fluctuating electric fields is charge- and mass-independent, without RMP the ambipolarity condition $\Gamma_{\perp, r}^{i}=\Gamma_{\perp, r}^{e}$ remains valid also when $\Gamma_{\perp, r}^{e, i}$ are enhanced by fluctuations above the neoclassical level. ${ }^{\frac{1}{23}}$

By combining Eqs. (5)-(9), one gets

$$
\Gamma_{\perp, r}^{i}=\Gamma_{\perp, r}^{e}-\frac{D_{\perp}^{i}}{D_{\perp}^{i}+D_{\|}^{e}} \Gamma_{\|, r}^{i}-D_{\perp, r}^{i} \frac{d n}{d r}+V_{\perp, r}^{i} n
$$

with

$$
\begin{aligned}
& D_{\perp, r}^{i}=\frac{D_{\perp}^{i} D_{\|}^{e}}{D_{\perp}^{i}+D_{\|}^{e}}\left(1+\frac{T_{i}}{T_{e}}\right), \\
& V_{\perp, r}^{i}=-\frac{D_{\perp, r}^{i}}{T_{e}+T_{i}}\left[\left(1-k_{\text {neo }}\right) \frac{d T_{i}}{d r}+\frac{1}{2} \frac{d T_{e}}{d r}+e B_{\vartheta} V_{\varphi}\right] .
\end{aligned}
$$

Here

$$
D_{\perp}^{i}=\sqrt{\frac{\pi}{2} T_{i} m_{i}} \frac{q T_{e}}{e^{2} B^{2} R}, D_{\|}^{e}=V_{\mathrm{th}, e} D_{F l}
$$

characterize the radial transfer of ions due to the deviation of their poloidal velocity from the neoclassical one and of electrons due to their motion along stochastic field lines, respec- tively. The effective perpendicular transport of ions is limited by the slowest of these processes. For a collisionally dominated plasma, the diffusivities $D_{\perp}^{i}$ and $D_{\|}^{e}$ are directly related to the conductivities $\sigma_{\perp}$ and $\sigma_{e r g}$ introduced in Ref. 24. It is important to notice that the two-fluid effects accounted in Eqs. (11)-(13) cannot be taken into consideration in the framework of ideal or resistive MHD models. ${ }^{25}$ Finally, we assume as in Ref. $5 \Gamma_{\perp, r}^{e}=-D_{\perp}^{e} d n / d r$ with neoclassical diffusivity $D_{\perp}^{e}$ (Ref. 14) in the ETB and an anomalous gyro-Bohm one of $0.6 \mathrm{~m}^{2} / \mathrm{s}$ beyond ETB; this allows us to reproduce well the density profile without I-coils when $\Gamma_{\perp, r}^{i}=\Gamma_{\perp, r^{*}}^{e}$

For the poloidal velocity, one obtains

$$
V_{\vartheta}=V_{\text {neo }}+\frac{E_{\text {neo }}-E_{\text {amb }}}{B_{\varphi}} \frac{D_{\|}^{e}}{D_{\perp}^{i}+D_{\|}^{e}}
$$

with

$$
E_{\text {neo }}=\frac{1}{e}\left[T_{i} \frac{d \ln n}{d r}+\left(1-k_{\text {neo }}\right) \frac{d T_{i}}{d r}+e B_{\vartheta} V_{\varphi}\right] .
$$

With $E_{r}=E_{\text {neo }}$ the perpendicular electron and ion flows are ambipolar, as in the case without RMP when $D_{\|}^{e}, \Gamma_{\|, r}^{i}=0$. Conversely to $E_{\text {amb }}, E_{\text {neo }}$ is normally negative in the ETB region. Thus, $E_{\text {neo }} \neq E_{\text {amb }}$ and with magnetic field stochastization $V_{\vartheta}$ deviates from $V_{\text {neo }}$ providing changes in the perpendicular ion transport. The resulting radial electric field can be expressed as follows:

$$
E_{r}=\frac{E_{\mathrm{neo}} D_{\perp}^{i}+E_{\mathrm{amb}} D_{\|}^{e}}{D_{\perp}^{i}+D_{\|}^{e}} .
$$

With increasing stochastization $E_{r}$ changes from the negative $E_{\text {neo }}$ to the positive $E_{\text {amb }}$. This is in qualitative agreement with the experimentally observed evolution of $E_{r}$ by activating the I-coils. ${ }^{12}$ A quantitative comparison of the model prediction and measured $E_{r}$ will be done elsewhere.

In stationary state, the radial density profile is calculated from the particle flux continuity equation,

$$
\Gamma_{r}=J,
$$

where $J$ is the density of the radial influx of recycling neutral particles. The transport of neutrals is described in the diffusion approximation by taking into account that the rate coefficient for charge exchange with ions, $k_{\mathrm{cx}}$, exceeds that for ionization by electrons, $k_{i}$. The corresponding transport equations, see, e.g., Ref. 26, are solved analytically by the WKB method, providing in the first approximation

$$
J(r) \approx J(a) \exp \left[-g \int_{r}^{a} n \sqrt{k_{i}\left(k_{i}+k_{\mathrm{cx}}\right) m_{i} / T_{i}} d r\right] .
$$

The geometric factor $g$ takes into account that neutrals enter the confined volume close to the $\mathrm{X}$ point where the distance between adjacent magnetic surfaces is much larger than on an average. ${ }^{27}$ 


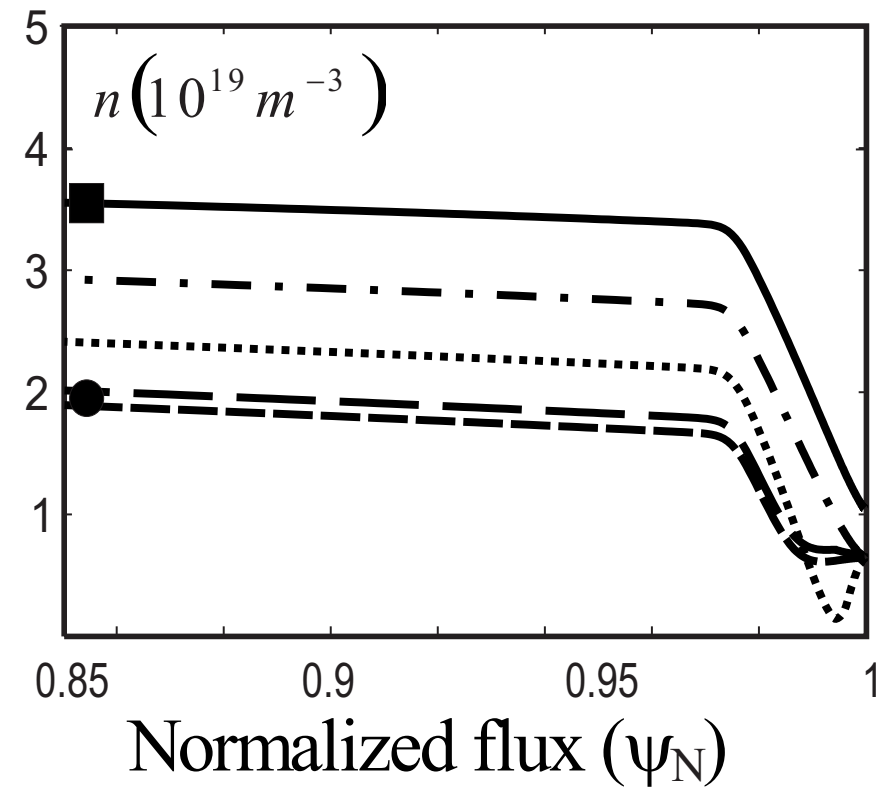

FIG. 1. Radial density profiles for DIII-D conditions computed without (solid curve) and with RMP from the I-coils by taking into account contributions from both parallel and perpendicular particle flows (short-dashed curve), with perpendicular (long-dashed curve) and parallel (dotted curve) contributions only, and with modified temperature profiles but without any changes in transport (dash-dotted curve).

\section{RESULTS OF CALCULATION}

In this study, we examine the particle transport and calculate the radial density profile across the plasma edge by using the electron and ion temperatures and toroidal rotation velocity from measurements. ${ }^{2,12}$ These data provide a toroidal velocity of $50 \mathrm{~km} / \mathrm{s}$ for carbon VI in the ETB. Estimates show that due to Coulomb collisions, carbon velocity cannot be very different from $V_{\varphi}$ for the main ions. One gets roughly the same level for the poloidal velocity by estimating $V_{\vartheta} \sim V_{\text {neo }} \sim 1 / e B_{\varphi} \times d T_{i} / d r$. Thus the terms with the toroidal velocity in Eqs. (8), (12), and (15) are of $B_{\vartheta} / B_{\varphi} \times d T_{i} / d r$ and contribute much less than others because $B_{\vartheta} \ll B_{\varphi}$.

The following parameters, characteristic for DIII-D deuterium discharges with the I-coil current of $3 \mathrm{kA},{ }^{2}$ have been used in calculations: the major and effective minor radius of the separatrix $R=1.67 \mathrm{~m}$ and $a=0.61 \mathrm{~m}$; the toroidal magnetic field $B=2 \mathrm{~T}$; the characteristic safety factor $q=4$ at the reference magnetic surface with the normalized flux $\psi_{\mathbf{N}} \equiv(r / a)^{2}=0.95$; the width of the electron transport barrier where without RMP the particle transport is reduced to the neoclassical level, $\Delta_{b}=0.92 \mathrm{~cm}$; the vacuum, i.e., without screening accounted, amplitude of RMPs with $M / N=(11-13) / 3$ provides $b_{M, N}=2.6 \times 10^{-4}$ at $\psi_{\mathrm{N}}=0.95 .^{2}$ The density of neutral influx through the separatrix is fixed at the same level as before RMP application, $J(a)=4.5$ $\times 10^{19} \mathrm{~m}^{-2} \mathrm{~s}^{-1}$, and $g=4 .^{5}$

Figure 1 shows the reference density profile versus $\psi_{\mathbf{N}}$ computed without RMP, solid curve, and those with RMP obtained under different assumptions about the composition of the radial particle flux $\Gamma_{r}$. Experimental data from Ref. 2 are presented by the filled box and circle, respectively. The short-dashed curve has been found with both perpendicular

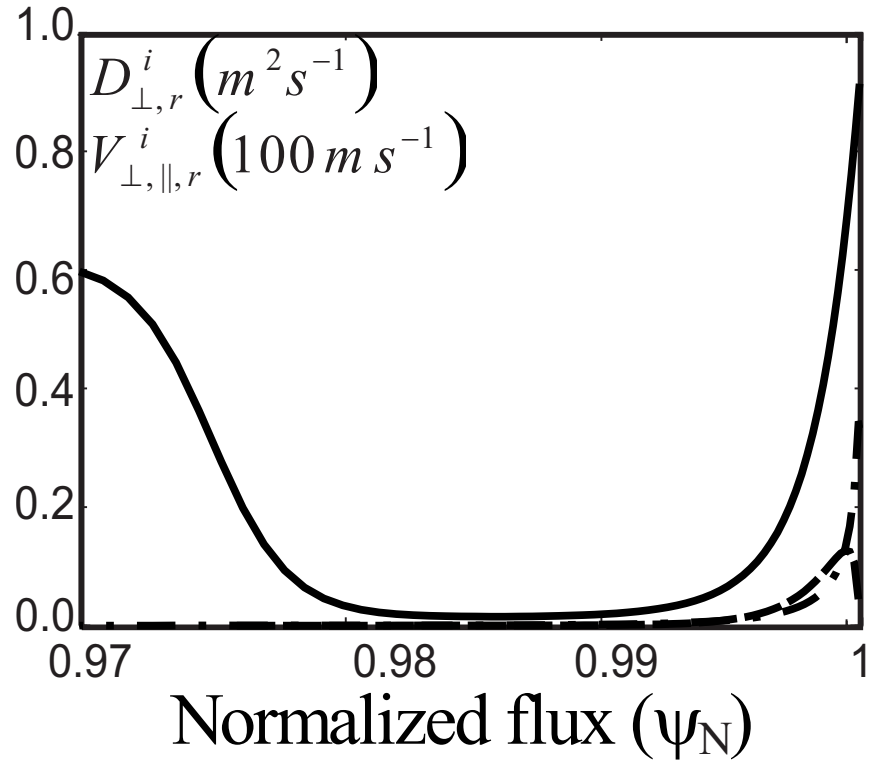

FIG. 2. Radial profiles of $D_{\perp, r}^{i}$ (solid curve), $V_{\perp, r}^{i}$ (dashed curve), and $V_{\|, r}^{i}=\Gamma_{\|, r}^{i} / n$ (dash-dotted curve).

and parallel contributions, $\Gamma_{\perp, r}^{i}$ and $\Gamma_{\|, r}^{i}$, and reproduces well the experimentally found plasma density at the reference position $\psi_{\mathrm{N}}=0.85, n_{0.85} \approx 1.93 \times 10^{19} \mathrm{~m}^{-3}$. A bit higher $n_{0.85}$ follows if $\Gamma_{\perp, r}^{i}$ only is taken into account, see the longdashed curve. However, with $\Gamma_{\perp, r}^{i}$ neglected but $\Gamma_{\|, r}^{i}$ retained (dotted curve), the computed density is significantly higher and the pump out effect is much lower than observed experimentally. It is even weaker (see the dash-dotted curve) if no changes in transport at all but only the experimentally found modification of the ion and electron temperatures are taken into account. Thus, under the conditions in question, the perpendicular ion transfer is more important than the parallel transport. The radial profiles of the transport characteristics $D_{\perp, r}^{i}, V_{\perp, r}^{i}$, and $V_{\|, r}^{i}=\Gamma_{\|, r}^{i} / n$ are shown in Fig. 2. With $\Gamma_{\perp, r}^{i}$ and $\Gamma_{\|, r}^{i}$ taken into consideration, the model proposed reproduces well enough also the density profile obtained with the I-coil current of $2 \mathrm{kA} .^{2}$

As was already mentioned above, the question of the RMP screening is a very difficult one and is one of the main sources of uncertainty in our modeling. Therefore, we have studied the sensitivity of the results to the screening factor by varying its radial dependence, as is shown in Fig. 3(a), where the solid curve reproduces the radial dependence from Fig. 5 of Ref. 7. Figure 3(b) displays the corresponding calculated density profiles. One can see that the density profile is sensitive enough to the screening factor. However, in reality there is probably not too much freedom to vary arbitrarily the screening factor because changes in the plasma density have a feedback reaction on $f_{\text {scr }}$. Indeed, decreasing density results through the modification in the perpendicular heat conduction in increasing temperature. ${ }^{4,5}$ Therefore, the parallel plasma resistivity, essentially affecting RMP screening process, reduces and $f_{\text {scr }}$ drops. In order to take this into account, all processes involved, i.e., particle and heat transport and penetration of MRP, have to be modeled in the future self-consistently. 


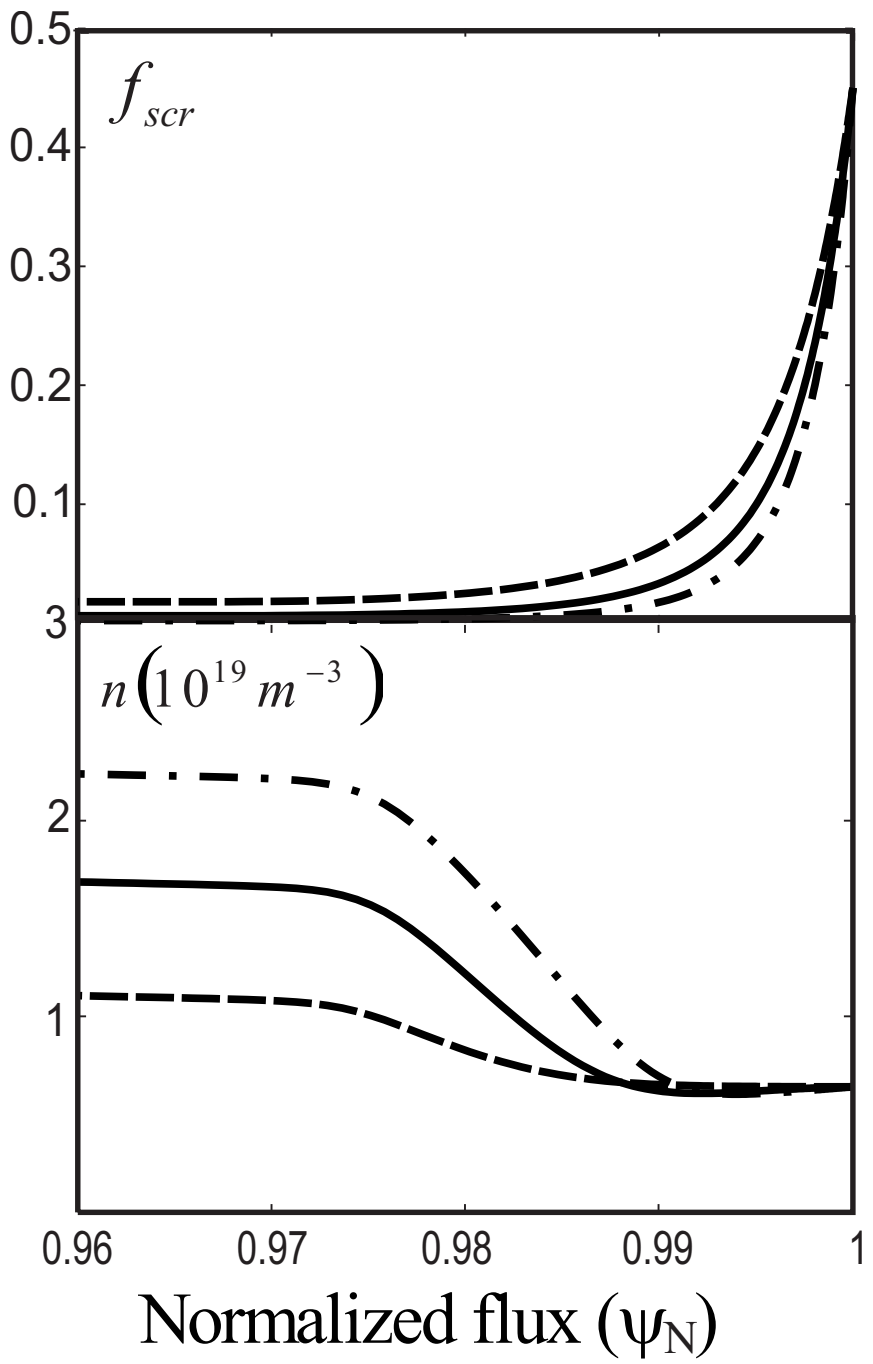

FIG. 3. Radial profiles of the screening factor assumed in the present computations, with the solid curve corresponding to the results of Ref. 7 (a) and calculated density profiles (b).

Alternatively to the transport processes discussed above, some rise of the electron perpendicular transport due to increasing fluctuation level, observed experimentally with $\mathrm{RMP}^{28}$ may be of importance. In order to analyze its relevance to the pump out, $D_{\perp}^{e}$ has been increased at the separatrix by the factor $\gamma \geqslant 1$ above the neoclassical level $D_{\perp}^{\text {neo; }}$ inside the electron ETB, the enhancement degree $\delta(r) \equiv D_{\perp}^{e} / D_{\perp}^{\text {neo }}-1$ was assumed varying with the local RMP magnitude,

$$
\delta(r)=(\gamma-1) \cdot\left|\widetilde{\mathbf{B}}_{r}(r) / \widetilde{\mathbf{B}}_{r}(a)\right|^{\xi} .
$$

Figure 4 demonstrates $n_{0.85}$ versus $\gamma$ and one can see that for linear scaling, $\xi=1$, the experimental level of the particle pump can be achieved with $\gamma \approx 40$. For quadratic dependence, $\xi=2$, which is normally predicted by theory (see, e.g., Ref. 29), only a much higher enhancement in the electron perpendicular transport could bring a noticeable effect. The strong increase of $D_{\perp}^{e}$ required to reproduce the experimental pump-out level would mean a corresponding increase of the electron perpendicular heat conductivity up to a level critical for the ETB existence. Therefore, it is probably unrealistic to

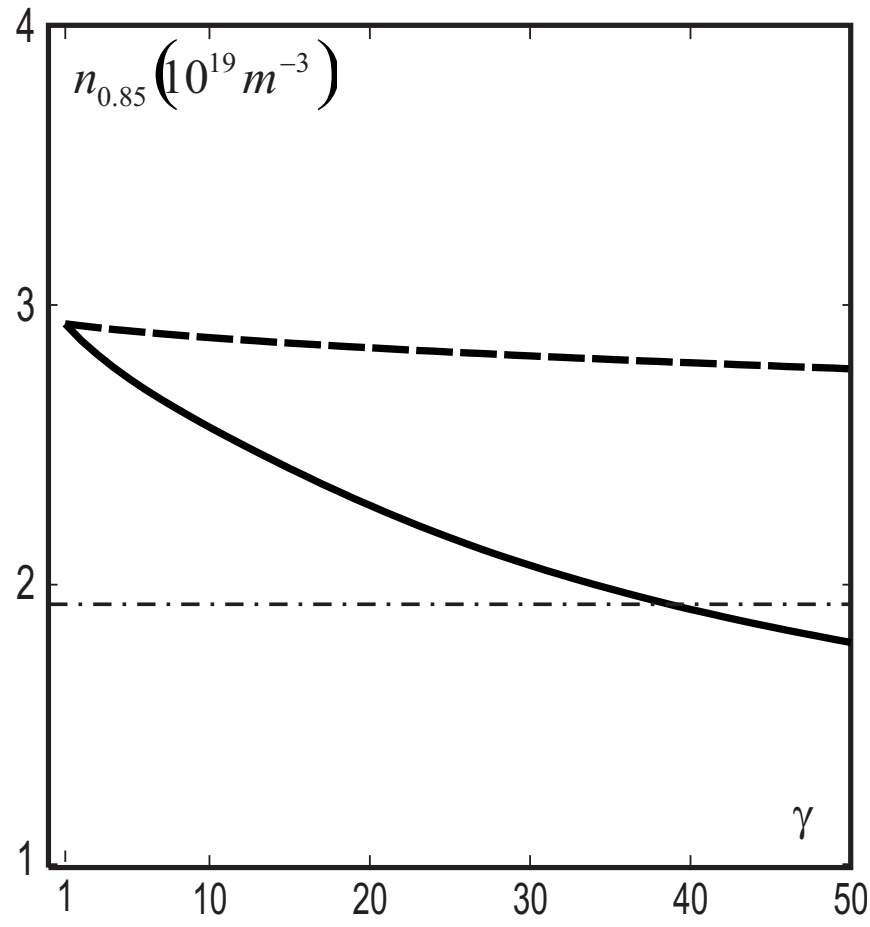

FIG. 4. The $\gamma$ dependence of $n_{0.85}$ computed under assumptions that inside the electron ETB either $\xi=1$ (solid curve) or $\xi=2$ (dashed curve), and compared with the experimental level $n_{0.85} \approx 1.93 \times 10^{19} \mathrm{~m}^{-3}$ (dash-dotted line).

expect that the increase in $D_{\perp}^{e}$ is decisive for the density drop with RMP.

The factor $g$ in Eq. (18) depends in a complex way on the magnetic geometry, and $g=4$ assumed above is only a rough estimate. Therefore, we have examined the sensitivity of the results to this parameter. In particular, $n_{0.85}$ is decreased by $5 \%$ only when $g$ is increased from 2 to 8 . This is explained by the fact that at relatively low plasma density in the experiments in question, the electron ETB is transparent for recycling neutrals so that the exponential factor calculated for $r=a-\Delta_{b}$ is close to 1 in the whole $g$ range considered. Beyond the electron ETB, the radial variation of $J$ is of minor importance for the density profile behavior since $n(r)$ is always flat due to high anomalous $D_{\perp}$.

\section{ACKNOWLEDGMENTS}

This study has been partially supported by the International Bureau of the German Ministry for Education and Research, Project IND 06/24, and by the U.S. Department of Energy, Contract No. DE-FC02-04ER54698.

${ }^{1} \mathrm{Ph}$. Ghendrih, A. Grosman, and H. Capes, Plasma Phys. Controlled Fusion 38, 1653 (1996)

${ }^{2}$ T. E. Evans, R. A. Moyer, K. H. Burrell, M. E. Fenstermacher, I. Joseph, A. W. Leonard, T. H. Osborne, G. D. Porter, M. J. Schaffer, P. B. Snyder, P. R. Thomas, J. G. Watkins, and W. P. West, Nat. Phys. 2, 419 (2006).

${ }^{3}$ Y. Liang, H. R. Koslowski, P. R. Thomas, E. Nardon, B. Alper, P. Andrew, Y. Andrew, G. Arnoux, Y. Baranov, M. Bécoulet, M. Beurskens, T. Biewer, M. Bigi, K. Crombe, E. De La Luna, P. de Vries, W. Fundamenski, S. Gerasimov, C. Giroud, M. P. Gryaznevich, N. Hawkes, S. Hotchin, D. Howell, S. Jachmich, V. Kiptily, L. Moreira, V. Parail, S. D. Pinches, E. Rachlew, and O. Zimmermann, Phys. Rev. Lett. 98, 265004 (2007). 
${ }^{4}$ M. Z. Tokar, T. E. Evans, A. Gupta, R. Singh, P. Kaw, and R. C. Wolf, Phys. Rev. Lett. 98, 095001 (2007).

${ }^{5}$ M. Z. Tokar, T. E. Evans, A. Gupta, D. Kalupin, A. Nicolai, R. Singh, and B. Unterberg, Nucl. Fusion 48, 024006 (2008).

${ }^{6}$ R. Fitzpatrick, Phys. Plasmas 5, 3325 (1998).

${ }^{7}$ M. F. Heyn, I. B. Ivanov, S. V. Kasilov, W. Kernbichler, I. Joseph, R. A. Moyer, and A. M. Runov, Nucl. Fusion 48, 024005 (2008).

${ }^{8}$ R. W. Harvey, M. G. McCoy, J. Y. Hsu, and A. A. Mirin, Phys. Rev. Lett. 47, 102 (1981).

${ }^{9}$ I. Kaganovich and V. Rozhansky, Phys. Plasmas 5, 3901 (1998).

${ }^{10} \mathrm{~V}$. Rozhanski and M. Tendler, in Reviews of Plasma Physics edited by B. B. Kadomtsev (Consultants Bureau, New York, 1991), Vol. 19, p. 147.

${ }^{11}$ H. A. Claassen, H. Gerhauser, A. Rogister, and C. Yarim, Phys. Plasmas 7, 3699 (2000).

${ }^{12}$ K. H. Burrell, T. E. Evans, E. J. Doyle, M. E. Fenstermacher, R. J. Groebner, A. W. Leonard, R. A. Moyer, T. H. Osborne, M. J. Schaffer, P. B. Snyder, P. R. Thomas, W. P. West, J. A. Boedo, A. M. Garofalo, P. Gohil, G. L. Jackson, R. J. La Haye, C. J. Lasnier, H. Reimerdes, T. L. Rhodes, J. T. Scoville, W. M. Solomon, D. M. Thomas, G. Wang, J. G. Watkins, and L. Zeng, Plasma Phys. Controlled Fusion 47, B37 (2005).

${ }^{13}$ T. E. Evans, K. H. Burrell, M. E. Fenstermacher, R. A. Moyer, T. H. Osborne, M. J. Schaffer, W. P. West, L. W. Yan, J. A. Boedo, E. J. Doyle, G. L. Jackson, I. Joseph, C. J. Lasnier, A. W. Leonard, T. L. Rhodes, P. R. Thomas, J. G. Watkins, and L. Zeng, Phys. Plasmas 13, 056121 (2006). ${ }^{14}$ J. Wesson, Tokamaks, 3rd ed. (Clarendon Press, Oxford, 2004).

${ }^{15}$ A. B. Rechester and M. N. Rosenbluth, Phys. Rev. Lett. 40, 38 (1978).

${ }^{16}$ A. I. Morozov and L. S. Solov'ev, Reviews of Plasma Physics, editted by M. A. Leontovitch (Consultants Bureau, New York, 1966), Vol. 2, p. 1.

${ }^{17}$ M. Bécoulet, E. Nardon, G. Huysmans, W. Zwingmann, P. Thomas, M. Lipa, R. Moyer, T. Evans, V. Chuyanov, Y. Gribov, A. Polevoi, G. Vayakis, G. Federici, G. Saibene, A. Portone, A. Loarte, C. Doebert, C.
Gimblett, J. Hastie, and V. Parail, Nucl. Fusion 48, 024003 (2008).

${ }^{18}$ M. J. Schaffer, J. E. Menard, M. P. Aldan, J. M. Bialek, T. E. Evans, and R. A. Moyer, Nucl. Fusion 48, 024004 (2008).

${ }^{19}$ A. Cole and R. Fitzpatrick, Phys. Plasmas 5, 3325 (1998).

${ }^{20}$ H. Capes, A. Samain, Ph. Ghendrih, and F. Nguyen, Contrib. Plasma Phys. 32, 192 (1992).

${ }^{21}$ A. Samain, H. Capes, Ph. Ghendrih, and F. Nguyen, Phys. Fluids B 5, 471 (1993).

${ }^{22}$ M. Z. Tokar, Phys. Plasmas 6, 2808 (1999).

${ }^{23}$ A. L. Rogister, Phys. Rev. Lett. 81, 3663 (1998).

${ }^{24}$ B. Unterberg, C. Busch, M. de Bock, J. W. Coenen, K. H. Finken, St. Jachmich, M. W. Jakubowski, Y. Kikuchi, A. Krämer-Flecken, M. Lehnen, U. Samm, O. Schmitz, S. Soldatov, M. Z. Tokar, M. von Hellermann, R. C. Wolf, Y. Xu, and TEXTOR-team, J. Nucl. Mater. 363-365, 698 (2007).

${ }^{25}$ E. Nardon, M. Bécoulet, G. Huysmans, and O. Czarny, Phys. Plasmas 14, 092501 (2007)

${ }^{26}$ M. Z. Tokar, Phys. Rev. Lett. 91, 095001 (2003).

${ }^{27}$ M. A. Mahdavi, R. Maingi, R. J. Groebner, A. W. Leonard, T. H. Osborne, and G. Porter, Phys. Plasmas 10, 3984 (2003).

${ }^{28}$ R. A. Moyer, K. H. Burrell, T. E. Evans, M. E. Fenstermacher, P. Gohil, I. Joseph, T. H. Osborne, M. J. Schaffer, P. B. Snyder, J. G. Watkins, L. R. Baylor, M. Bécoulet, J. A. Boedo, N. H. Brooks, E. J. Doyle, K.-H. Finken, P. Garbet, R. J. Groebner, M. Groth, J. Harris, E. M. Hollmann, G. L. Jackson, M. Jakubowski, T. C. Jernigan, S. Kasilov, C. J. Lasnier, A. W. Leonard, M. Lehnen, J. Lönnroth, E. Nardon, V. Parail, G. D. Porter, T. L. Rhodes, D. L. Rudakov, A. Runov, O. Schmitz, R. Schneider, D. M. Thomas, P. Thomas, G. Wang, W. P. West, L. Yan, J. H. Yu, and L. Zeng, Proceedings of the 21st IAEA Conference on Fusion Energy, 2006, Chengdu, edited by G. Mank and A. Malaquias (IAEA, Vienna, 2007), p. EX/9-3.

${ }^{29}$ R. E Waltz, Phys. Fluids 25, 1269 (1982). 